\title{
Erratum to: Environmental management practices in the Lebanese pharmaceutical industries: implementation strategies and challenges
}

\author{
May A. Massoud • N. Makarem • \\ Wijdan H. Ramadan • R. Nakkash
}

Published online: 29 January 2016

(C) Springer International Publishing Switzerland 2016

Erratum to: EnvironMonit Assess (March 2015)

187, Issue 3:4290

DOI 10.1007/s10661-015-4290-3

The original article contain an error.

The name of the third author should have been Wijdan H. Ramadan not Wijdan Ramadan. The corrected author name is shown above.

The online version of the original article can be found at http://dx. doi.org/10.1007/s10661-015-4290-3.

M. A. Massoud $(\bowtie) \cdot$ N. Makarem

Department of Environmental Health, Faculty of Health Sciences, American University of Beirut, P.O. Box 11-0236, Riad el Solh, Beirut 1107 2020, Lebanon

e-mail: maymassoud@yahoo.com

W. H. Ramadan

School of Pharmacy, Lebanese American University, Beirut,

Lebanon

R. Nakkash

Department of Health Promotion and Community Health, Faculty of Health Sciences, American University of Beirut, Beirut,

Lebanon 